\title{
Fluxes of sediment beneath floating silt screens due to density gradients and screen motion
}

\author{
Aschmoneit, Fynn Jerome; Hjelmager Jensen, Jacob; Saremi, Sina; Hélix-Nielsen, Claus
}

Published in:

Journal of Waterway, Port, Coastal, and Ocean Engineering

Publication date:

2020

Document Version

Peer reviewed version

Link back to DTU Orbit

Citation (APA):

Aschmoneit, F. J., Hjelmager Jensen, J., Saremi, S., \& Hélix-Nielsen, C. (2020). Fluxes of sediment beneath floating silt screens due to density gradients and screen motion. Journal of Waterway, Port, Coastal, and Ocean Engineering, 146(4), [04020010].

\section{General rights}

Copyright and moral rights for the publications made accessible in the public portal are retained by the authors and/or other copyright owners and it is a condition of accessing publications that users recognise and abide by the legal requirements associated with these rights.

- Users may download and print one copy of any publication from the public portal for the purpose of private study or research.

- You may not further distribute the material or use it for any profit-making activity or commercial gain

- You may freely distribute the URL identifying the publication in the public portal 


\title{
Fluxes of sediment beneath floating silt screens due to density
}

\author{
gradients and screen motion
}

\author{
Fynn Aschmoneit, Jacob Hjelmager Jensen† Sina Saremi ${ }^{\ddagger}$ and Claus Hélix-Nielsen ${ }^{\S}$
}

October 27, 2019

sediment transport, silt screen, gravity currents, screen motion

\section{Abstract}

4 Floating silt screens are widely applied to confine and control turbidity plumes and sedimentation due to dredg-

5 ing activities. Their efficient deployment requires detailed knowledge of the ambient hydrodynamic conditions.

6 Incorrect deployment may lead to confinement deficiencies, screen- burial or damage. One aspect of deployment

7 efficiency is the sediment transport rate beneath the silt screen. The sediment transport between the sides of a

8 floating silt screen is mostly affected by ambient currents. Besides, gravity currents and shear- stress induced

9 screen motion also affect the sediment transport. The gravity current is governed by the suspended sediment 


\section{Introduction}

Dredging activities have a considerable impact on aquatic ecosystems. Especially the resultant sedimentation and turbidity are harmful to many species. Sessile or immobile organisms are primarily endangered by burial through sedimentation, (Erftemeijer and Lewis, 2006). Increased turbidity fatally affects seagrass and photosynthetic symbionts, such as algae, through reduced light transmission, inhibiting photosynthesis, and therefore disrupting the aquatic food chain, (Fraser et al., 2017), ( Erftemeijer, Riegl, Hoeksema, and Todd, 2012).

Containment efforts to minimise the effects on marine ecosystems involve the implementation of environmental windows, forbidding dredging activities during sensitive periods, such as during fish- or coral spawning seasons (Fraser et al., 2017). Another way of dredging impact containment is to confine the spread of sedimentation and turbidity locally.

Floating silt screens are elastic barrier curtains, that extend from the water surface down to a specific depth. They are deployed in such a manner as they enclose sediment plumes or turbid water, thereby allowing controlled, local sedimentation, which leads to an increasing mud layer on the sea bed. The curtain extends so deep to leave a gap above the sea bed or mud layer which allows turbid water to disperse into the sea water. This turbidity dispersion can be controlled through optimal deployment of the curtain, which requires careful considerations of the ambient hydrodynamic conditions, see Fig. 1. Silt screens are commonly used at dredging and reclamation sites. Other application examples include the control of sediment plumes at harbour constructions sites or at industrial water intakes (WU, Neo, Jain, and Tan, 2016). Their effectiveness is defined through the relative turbidity reduction outside the enclosed area. Effective silt screen applications may yield a turbidity reduction in the ambient water of up to $90 \%$, (JBF Scientific Corporation, 1978).

Silt screen construction or deployment have been addressed in scientific articles since the late 1970s. An extensive theoretical analysis of ideal screen design, including the screen material, its buoyant swimmers and the screen connectors, and the screen deployment, in terms of screen depth, was presented in (JBF Scientific Corporation, 1978). This analysis concentrated on estimating the screen strain in different operating- and deployment conditions, and not on the actual turbid water transport. Vu et al. (Vu and Tan, 2010) presented experimental work on silt screen deformation for perpendicularly inciding flow (cross current), concluding that the screen deployment affects the flow distribution, which might even lead to sediment entrapping. In (WU et al., 2016), a numerical analysis of the screen deployment configuration around a water intake was presented. However, this two-dimensional study did not investigate the effect of ambient currents or the silt screen depth. Yasui et al. (Yasui, Deguchi, and Ono, 1999) defined the performance of screen deployment by the turbidity flux, for 


$$
j_{\perp}=\frac{\left\langle\left|\dot{V}_{t}\right|+\left|\dot{V}_{s}\right|\right\rangle_{T}}{A_{\Omega}}
$$

67 The turbid flow fraction $\alpha$ is a measure for the relative amount of turbid flow to total gross flow exchange

$$
\alpha=\frac{\left|\dot{V}_{t}\right|}{\left|\dot{V}_{t}\right|+\left|\dot{V}_{s}\right|}
$$


It is the aim of the Gravity Current Model to quantify how the isolated effect of buoyancy induced gross volume flux scales with the suspended sediment concentration and the screen depth. Likewise, the Harmonic Motion Transport Model examines the gross volume flux, induced by screen motion, in dependence of ambient parallel flow and the screen depth. For both transport mechanisms, characteristic turbid flow fractions are formulated. Note, that neither of the two models includes particle settling. Since the time scale involved in the settling of silt or clay is magnitudes smaller than the time scale involved in the water passing under the screen in both models, particle settling can safely be disregarded in this study. Nevertheless, the effect of the sedimentation induced sea bed increase is integrated through the analyses of screen depths.

First, the Gravity Current Model is presented. This covers the governing model theory, its implementation, and the model results. Thereafter, the Harmonic Motion Transport Model is presented, which covers the theory for the screen dynamics, the numerical model, and the derived results. Finally, the derived transport quantification relations and their applicability for screen deployment guidelines are discussed.

Both computational models were implemented in the open source computational fluid dynamics package OpenFOAM and executed on the high performance computing facility at the Technical University of Denmark. The open development of OpenFOAM allows for the implementation of custom solvers or boundary conditions, which has been done for both presented models. Additionally, MIKE FM, a state-of-the-art hydraulic modelling tool being used extensively in the engineering community, was employed on the Gravity Current Model. MIKE FM is capable of simulating density currents and was therefore chosen as a reference software.

\section{Gravity Current Model}

Two reservoirs of sea water and turbid water are separated through a stiff silt screen down to a specific height, leaving a gap above the seabed. Due to the different densities of the water bodies, an up-streaming sea water plume develops on the turbid water side, while a creeping plume of turbid water develops along the seabed on the sea water side, see Fig. 2. Hence, the resultant gross volume flux is solely a gravity current. The buoyant driving force is governed by the density gradient and the size of the plumes themselves. The water density and the plume sizes, in turn, are governed by the suspended sediment concentration and the screen depth, respectively. This isolated transport mechanism is symmetric with respect to the lateral direction and can therefore be analyzed through a two-dimensional model. In this model the gravity current is quantified for the turbidity concentration and the screen gap height. 


\subsection{Model Description}

The buoyancy driven water transport is dominated by the density difference of the sea water and the turbid water. The local fluid density $\rho$ is defined through the sea water density $\rho_{w}$, the volumetric sediment concentration $c$ and the sediment density $\rho_{s}$ :

$$
\rho=c \rho_{s}+(1-c) \rho_{w}
$$

The Boussinesq approximation for buoyancy driven flow applies when flow can be assumed incompressible but buoyancy effects are present. It simply assumes that the inertia terms in the Navier-Stokes equations are unaffected by density variations, however, density variations exert an external force on the fluid. Hence, the variable fluid density $\rho$ appears only in the gravity term:

$$
\begin{aligned}
\rho_{w}\left(\partial_{t} u_{i}+u_{j} \partial_{j} u_{i}\right) & =-\partial_{i} p+\mu \partial_{j j} u_{i}+\rho g_{i} \\
& =-\partial_{i} P+\mu \partial_{j j} u_{i}+c\left(\rho_{s}-\rho_{w}\right) g_{i}
\end{aligned}
$$

where $u_{i}$ is the $i$-th velocity component, $p$ is the pressure, $\mu=1 \mathrm{mPas}$ is the fluid viscosity and $g$ is the gravitation constant. The second line incorporates the substitution $P=p+\rho_{w} x_{j} g_{j}$, which conveniently separates the buoyant force from the hydrostatic pressure. Because of the low Reynolds number of the buoyant plumes, the flow is assumed laminar.

The Boussinesq approximation is favored over two-phase solvers, as the marginal density difference of the water bodies does not create a significant surface tension effect. The approximation is also preferred over fully mixing solvers, due to its greater numerical performance, while producing similar results for the considered model.

Sedimentation and diffusion are not considered in this model. They are much slower transport mechanisms and thus, these mechanisms are isolated from the buoyant transport in this study. The silt concentration is therefore only advected with the velocity field $u$ :

$$
\partial_{t} c=-u_{j} \partial_{j} c
$$

Finally, total mass is conserved through the continuity equation: 


$$
\partial_{j} u_{j}=0
$$

The system is initialised with two unperturbed water bodies of turbid water and sea water. Along the screen gap the two bodies form a sharp interface. As the model is left to find its equilibrium state, two plumes of seaand turbid water arise at the interface. The light sea water plume rises upward along the screen, reaches the water surface and propagates along the water surface, while the heavy turbid plume creeps along the sea bed. The flow reaches an oscillating equilibrium as the plumes leave the domain. It is oscillatory, because the stiff screen bottom edge promotes small eddies as flow is forced by and shear flow of the plumes with the respective bulk reservoir cause Kelvin-Helmholtz instabilities.

\subsection{Implementation}

The model is implemented in a 2-dimensional, rectangular domain of height $d_{s b}$ and width $2 \Delta_{x}$, in which the silt screen is placed in the domain middle. All applied boundary conditions are either of Neumann type, Dirichlet type, or a combination of these, see Fig. 2 for boundary naming convention. The most significant boundary conditions are defined on the $X 0$ and $X 1$ edges, as the fluid must be able to both, enter, and leave the domain along these edges. Neumann conditions were therefore applied for the velocity. This allows to define dependent boundary conditions for the concentration field: They are of Neumann-type for areas of outflow, while they act as Dirichlet boundary conditions, with the respective bulk concentration value, for inflow. All applied boundary conditions are summarised in Table 1.

OpenFoam's implementation of a buoyancy driven flow with the Boussinesq approximation, 'buoyantBoussinesqPimpleFoam', was modified to simulate concentration dependent buoyancy, i.e. the density difference is calculated through $\rho^{\prime}=c\left(\rho_{s}-\rho_{w}\right)$.

A domain width analysis yielded stable results for $\Delta_{x} \geq 50 \mathrm{~m}$. The domain was discretized in a regular, rectangular mesh, with an increasing density towards the edges $Y 0, Y 1$ and $S C$. A mesh resolution analysis was conducted through the Grid Convergence Index (GCI) (Roache, 1998), which states the relative difference to an infinitely finely resolved mesh, through comparing results from successively refined meshes. The GCI test yielded a value of $1.3 \%$ on the total flow rate under the screen, for an average discretization of $20 \frac{\mathrm{cells}}{\mathrm{m}}$. 


\subsection{Results}

Table 2 summarizes the set of model parameters, in which the italic font indicates those parameters, which the gravity current is quantified against.

A snap shot of a simulation is shown in Fig. 3. The turbidity concentration is indicated by the red colour intensity and the flow currents can be seen though the black arrows. The creeping turbid water plume and the buoyant sea water plume are forced beneath the silt screen. Due to their opposing direction of motion, shear forces along the sea water - turbid water - interface cause Kelvin-Helmholtz instabilities, which are marked in Fig. 3 with green ellipses. As the opposing flows passing each other beneath the screen have reached an equilibrium state, the magnitudes of the two plumes' flow rates are same. That defines the turbid flow fraction in equation (7) for this model as $\alpha=0.5$.

Since this is a two-dimensional model, the void area in equation (1), $A_{\Omega}$, is reduced by one dimension and defined through the screen gap $\delta$. This defines the gravity current as:

$$
j_{\perp}=\frac{\left\langle\left|\dot{V}_{t}\right|+\left|\dot{V}_{s}\right|\right\rangle_{T}}{\delta}
$$

The gravity current is expressed through the Froude number, using the reduced gravity $g^{\prime}=g\left(1-\rho_{w} / \rho\left(c_{s}\right)\right)$, where $\rho\left(c_{s}\right)$ is the bulk turbid water density:

$$
F r=\frac{j_{\perp}}{\sqrt{g^{\prime} \delta}}
$$

Figures 4 and 5 show the simulation results of the gravity current in dependence of the turbidity concentration and the screen gap, respectively.

From the results show in Fig. 4, it can be seen that the flow patterns are well characterized by the Froude number scaling. Nevertheless, weak variations with respect to both, the concentration and the gap height are detected. These are attributed to mesh deficiencies or the inaccuracy of the data extraction with higher cross flow velocities.

Fig. 5 shows the gravity current, in terms fo the Froude number, against the non-dimensional screen gap $\hat{\delta}=\delta / d_{s b}$. The data does not show a significant dependence of the Froude number on the screen gap, suggesting that the Froude number renormalisation of the turbid water flux, equation (8), describes the bottom gap 
dependence well. The simulations conducted with OpenFOAM and the four additional simulations conducted with MIKE 3, agree well, yielding a Froude number of $F r \sim 0.5$. The fluctuations are thought to be caused by boundary effects at the X0 and X1 boundaries, where inflow and outflow must be allowed on opposing sides simultaneously. If the silt screen was removed $(\hat{\delta}=1)$, the system would resemble a classical lock-exchange condition. For this set-up, Benjamin (Benjamin, 1968) derived a gravity current of $F r=0.5$, in the limit of total energy conservation. The lock-exchange gravity current is included in Fig. 5 as a yellow cross, which is very similar to the simulated silt screen gravity currents.

The scaling analysis showed that the gravity current under the silt screen is similar to a lock-exchange and can be well described by a Froude number of $F r=0.5$. Based on this, the total sediment mass flux per unit screen length, $\dot{M}_{s}^{\prime}$, can be evaluated through the turbid water volume flow rate per unit screen length, $\dot{V}_{t}^{\prime}$ :

$$
\begin{aligned}
\dot{M}_{s}^{\prime} & =\dot{V}_{t}^{\prime} c_{s} \rho_{s}=\alpha j_{\perp} \delta c_{s} \rho_{s} \\
& \approx \frac{1}{4} g^{\prime}\left(c_{s}, \rho_{s}\right)^{\frac{1}{2}} \delta^{\frac{3}{2}} c_{s} \rho_{s}
\end{aligned}
$$

Fig. 6 shows unit length mass flow against the volumetric sediment concentration, according to equation (9), illustrating the relevance of the gravity current. The mass flow was calculated for silt $\left(\rho_{s}=2800 \mathrm{~kg} / \mathrm{m}^{3}\right)$ and clay $\left(\rho_{s}=1200 \mathrm{~kg} / \mathrm{m}^{3}\right)$, and two different screen gap heights, visualizing that the gap height has a greater influence than the sediment density. Note that the graphs do not represent steady-state solutions: At constant sediment spill rate, the mass flow is constantly decreasing, due an increasing sea bed level, which decreases the screen gap, and due to sedimentation and dilution of the turbid water reservoir.

\section{Harmonic Motion Transport Model}

An elastic silt screen hanging in the streamwise direction of an ambient flow will be exposed to shear stress discontinuities across the screen, which lead to downstream propagating screen deflections. These deflections are reflected upstream at the moored end of the screen, which causes interference of opposing deflections, or, a 'flapping' motion. Thereby, the flapping screen motion is forcing the flow around the screen and promotes pumping of water under the screen. In clean water the net volume current is zero, but the instantaneous gross volume current depends on the ambient flow magnitude and the screen depth. The three-dimensional harmonic motion model quantifies the gross volume flux for these two parameters.

\subsection{Screen Motion Model}


The mutual influence of the ambient flow on the screen tissue and the resulting screen motion of the ambient flow is simplified in this model. A study of flag flapping in parallel flow shows oscillating, harmonic flag motions (Michelin, Llewekkyn Smith, and Glover, 2008). The situation of a silt screen in parallel flow is similar, with the exception, that the silt screen is constrained on both ends, in which case the oscillatory motion is a wave motion. Therefore, for simplicity, it is assumed that an imposed wave motion yields a similar gross volume flux as a shear-stress inflicted flapping motion.

Furthermore, because the silt screen is moored in place, the elastic screen is assumed immobile in the mooring planes, and along the float tube, see Fig. 1. In the following, $x$ denotes the horizontal downstream direction, with $x=0$ at the upstream mooring position, and $y$ denotes the downward direction, with $y=0$ at sea level, and the screen length $l_{s c}$. The boundary conditions for the deflection $\xi(x, y, t)$ can then be summarized as:

$$
\xi(0, y, t)=0 \quad, \quad \xi\left(l_{s c}, y, t\right)=0 \quad, \quad \xi(x, 0, t)=0
$$

Inspired by the work of Manela and Howe (Manela and Howe, 2009), who derived a stable standing wave solution for flag flapping in low Re flow, the elastic screen motion is modeled as two mutual opposing propagating waves, which interfere to an harmonic standing wave of amplitude $A(y)$, frequency $\omega$ and wave number $k$. The screen deflection $\xi(x, y, t)$ is therefore defined through:

$$
\xi(x, y, t)=A(y) \cos (\omega t) \sin (k x), \quad x \in\left[0, l_{s c}\right]
$$

In this section, a flapping motion theory is derived, which comprises of definitions for the oscillation amplitude, the oscillation wave length and frequency, based on the environment- and deployment conditions of the silt screen. The maximum oscillation amplitude $A_{0}$ and the oscillation mode $m$ remain open parameters, against which the gross volume flux is also quantified.

\section{Amplitude condition}

In order to satisfy the boundary conditions (10) the oscillation amplitude has to vanish as $y \longrightarrow 0$. An upwards linearly decreasing amplitude from $A_{0}$ at the screen depth $y=d_{s b}-\delta$ was chosen for this model: 


$$
A(y)=A_{0} \frac{y}{d_{s b}-\delta}, \quad y \in\left[0, d_{s b}-\delta\right]
$$

As an absolute upper limit for $A_{0}$ a tearing amplitude $A_{\mathrm{t}}$ is defined as the amplitude at which the screen material cannot withhold its inner stress and tears apart. The tearing stress is given by the material property tensile strength $\sigma_{\mathrm{ts}}$ and the deployment property mooring stress $\sigma_{0}$. The material property Young's modulus $E$ relates the stresses to the respective strains $\varepsilon_{0}, \varepsilon_{\mathrm{osc}}$. The tearing condition reads as:

$$
\sigma_{\mathrm{ts}} \leq \sigma_{0}+\sigma_{\mathrm{osc}}=E\left(\varepsilon_{0}+\varepsilon_{\mathrm{osc}}\right),
$$

The oscillation strain is defined through the relative difference of the curve path length and wave length $\lambda=\frac{2 \pi}{k}$ :

$$
\varepsilon_{\text {osc }}=\frac{\int_{0}^{\lambda} \sqrt{1+A_{0}^{2} k^{2} \cos ^{2}(k \phi)} d \phi-\lambda}{\lambda} \approx \frac{1}{2} A_{0}^{2} k^{2},
$$

assuming $A_{0} \ll \lambda$. Equations (13) and (14) define the tearing amplitude $A_{t}$, which must always be greater than the chosen oscillation amplitude $A_{0}$.

$$
A_{0} \stackrel{!}{<} A_{t}=\frac{\lambda}{\pi \sqrt{2}} \sqrt{\frac{\sigma_{\mathrm{ts}}}{E}-\varepsilon_{0}}
$$

\section{Wave Length condition}

As silt screens are usually applied in sets of connected screens the deflections must be continuous across individual screens. A periodic oscillation across the screen length satisfies that condition. This implies that the screen length must coincide with integer multiples of the wave length:

$$
\lambda=\frac{l_{s c}}{m} \quad, \quad m \in \mathbb{N} .
$$




\section{Oscillation Frequency condition}

Because of the screen's elasticity, it opposes deflections, minimizing its surface area, which is analogous to a molecular fluid-fluid interface. A surface tension $\gamma$ can therefore be defined for the screen, using the local stress $\sigma_{\text {loc }}$ and the screen thickness $d_{s c}$ :

$$
\gamma=\sigma_{\mathrm{loc}} \delta_{s c} \approx E \varepsilon_{0} d_{s c}
$$

For small oscillation amplitudes, $A_{0} \ll \lambda$, the local stresses can be assumed uniform and therefore equal to the mooring stress $\sigma_{0}$, justifying the above approximation (JBF Scientific Corporation, 1978).

The oscillation frequency $\omega$ can be defined as a function of the material properties, the environmental conditions water density $\rho_{w}$, sediment concentration $c$ and downstream flow $U_{\|}$, and the aforementioned surface tension $\gamma$, through an analogy to stable solutions of stratified flow. Analyses of the instability growth of perturbed interfaces in stratified flow show that stable interface motions are possible if gravitation or surface tension effects are included (Kelvin-Helmholtz instability). Interface motion solutions, with wave number $k^{\prime}$ and rate $\omega^{\prime}$, are given by $\xi \sim e^{i\left(k^{\prime} x-\omega^{\prime} t\right)}$, which describe propagating waves, if $\omega^{\prime}$ is real-valued, and exponentially growing instabilities, if $\omega^{\prime}$ is a complex. The dispersion relation of the Kelvin-Helmholtz instability is (Chandrasekhar, 1981):

$$
\omega^{\prime}=k^{\prime} \frac{\rho_{1} U_{1}+\rho_{2} U_{2}}{\rho_{1}+\rho_{2}} \pm\left[-\frac{k^{\prime 2} \rho_{1} \rho_{2}\left(U_{1}-U_{2}\right)^{2}}{\left(\rho_{1}+\rho_{2}\right)^{2}}+\frac{{k^{\prime}}^{3} \gamma-k^{\prime} g\left(\rho_{1}-\rho_{2}\right)}{\rho_{1}+\rho_{2}}\right]^{\frac{1}{2}},
$$

where $g$ is the gravitation constant, and $\rho_{1,2}$ are the densities of the respective streams. As can be seen from equation (18), the interface motion is stable if the term in the square root is positive and $\omega^{\prime}$ can be regarded as an angular frequency. Because gravity is omitted and the velocities are equal in this model $\left(U_{1}=U_{2} \doteq U_{\|}\right)$, the square root argument is always positive and $\omega^{\prime}$ is real-valued. The minus sign in front of the square root in (18) is omitted since $\omega^{\prime}$ would only be positive for $U_{\|} \gtrsim 2.5 \frac{\mathrm{m}}{\mathrm{s}}$, which exceeds the silt screen application limits and is therefore not considered here. Substituting $\omega^{\prime} \rightarrow \omega, k^{\prime} \rightarrow k$ and $\rho_{1,2} \rightarrow \rho_{w, t}$, the resultant expression for the screen oscillation frequency reduces to: 


$$
\omega=k U_{\|}+\left[\frac{k^{3} \gamma}{\rho_{w}+\rho_{t}}\right]^{\frac{1}{2}},
$$

The oscillation frequency thus consists of a linear term and a correction term, which scales with the square root of the surface tension. Note that $\lim _{U_{\|} \rightarrow 0} \omega \neq 0$, which is due to the surface tension effect. This implies for the oscillation amplitude that $\lim _{U_{\|} \rightarrow 0} A_{0}=0$.

\subsection{Model Description}

The harmonic motion transport model is centred around the implementation of the harmonic screen dynamics (11) in a CFD model. The computational domain is block-shaped of dimensions $\left(\lambda, \Delta_{y}, \Delta_{z}\right)$, see Fig. 7 . The surface pair $(X 0, X 1)$ is defined as periodic boundaries to allow periodicity of the system in downstream direction. Neumann boundary conditions are defined on all variables on the $(Y 0, Z 0, Z 1)$ surfaces. On $(Y 1, S C)$ only, the velocity boundary condition are of Dirichlet type, imposing a no-slip condition. Turbulence is modelled with the $k-\epsilon$ model and initial turbulent velocity fluctuation and characteristic length scale are set to $A_{0} \omega / 2 \pi$ and $0.1 \delta$, respectively.

The open source CFD software OpenFOAM was chosen for this model, as it allows the implementation of the screen dynamics through modifications of existing dynamic mesh libraries. For this model the analytic mesh motion algorithm dynamicInkJetFvMesh ("OpenFOAM", 2019) was modified to develop the oscillating mesh expansion/contraction algorithm dynamicExplicitMotion(Aschmoneit, 2018). This dynamic mesh algorithm displaces all mesh vertices in the $z$-direction through:

$$
\zeta_{z}\left(x, y, z_{0}, t\right)=\tau\left(z_{0}\right) \xi(x, y, t), \quad x \in[0, \lambda], \quad y \in\left[0, \Delta_{y}\right], \quad z_{0} \in\left[-\frac{\Delta_{z}}{2}, \frac{\Delta_{z}}{2}\right]
$$

The displacement follows the screen dynamics defined in equation (11) with an increased validity range for $y$ and the damping function $\tau$, which reduces the mesh displacement linearly with distance from the screen:

$$
\tau\left(z_{0}\right)=1-\frac{2\left|z_{0}\right|}{\Delta_{z}}
$$


undeformed. OpenFOAM's dynamic mesh interface ensures that momentum and mass are conserved as the dynamics mesh algorithm changes the mesh volume through periodic expansions or contractions.

\subsection{Implementation}

The domain dimensions must be chosen in such a way that boundary effects are not influencing the simulation results. The domain analysis was conducted for a high meshing density, which was adjusted downwards afterwards.

A domain width of $\Delta_{z}=4 \mathrm{~m}$ was chosen. Fig. 8 visualises the flow in two different depths: The eddies indicate that water is being transported under the screen, while the flow is not much affected above the screen gap. It is therefore not necessary to simulate the whole water body. A domain height of $\Delta_{y}=3 \delta$ was therefore chosen. The computational mesh consists of hexahedrons only, which are not always perfectly orthogonal, due to the mesh motion. The ideal average mesh discretization was found to be $\rho_{x}=10 \frac{\mathrm{cells}}{\mathrm{m}}, \rho_{y}=50 \frac{\mathrm{cells}}{\mathrm{m}}$ and $\rho_{z}=20 \frac{\mathrm{cells}}{\mathrm{m}}$, which yields a total cell number in the range $\left[10^{6}, 10^{7}\right]$. The mesh resolution is increased perpendicularly to Dirichlet boundary patches and in the region beneath the screen. The GCI test (Roache, 1998) was conducted on various, successively refined mesh resolutions, using the total cross flow as the convergence quantity. For the above mentioned resolution, it yielded a value of $1.2 \%$. A fine time stepping (Courant number $\approx 0.2$ ) was necessary to avoid simulation instabilities due to the dynamic mesh.

\subsection{Results}

Various simulations were conducted with respect to the downstream flow $u_{\|}$, the screen gap $\delta$ and the open parameters oscillation amplitude $A_{0}$ and oscillation mode $m$. The screen material properties refer to a polyamidetype screen. All parameters are summarized in Table 3.

A series of turbidity profiles at different oscillation instances is shows in Fig. 9. It is clearly seen that turbid water is pumped to the sea water side, while sea water is pumped in the other direction. It must be assumed that a fraction of the pumped turbid water volume is back-pumped through the screen motion and similarly, some fraction of sea water will be back-pumped. This defines an upper limit for the turbid water flux fraction of:

$$
\alpha=0.5
$$


The gross volume flux $j_{\perp}$ is non-dimensionalized through:

$$
\hat{\jmath}_{\perp}=2 \pi \frac{j_{\perp}}{A_{0} \omega}
$$

The dimensionless current $\hat{\jmath}_{\perp}$ is plotted (indicated by crosses) against the relative oscillation amplitude $A_{0} / d_{s b}$, the normalized downstream flow, $\hat{u_{\|}}=u_{\|} / \sqrt{g d_{s b}}$, the non-dimensional screen gap, $\hat{\delta}=\delta / d_{s b}$ and the oscillation mode $m$ in figures 10, 11, 12 and 13 .

Fig. 10 shows that the oscillation amplitude is not affecting the flux, under the renormalization (23), while the downstream flow and the oscillation mode do affect the flux. The downstream flow plot 11 indicate a scaling relation for the downstream magnitude. Likewise, the dependencies on the screen gap and the oscillation mode indicate scaling relations. It is therefore suggested to express the current through:

$$
\hat{\jmath}_{\perp} \sim \hat{\delta}^{\phi_{1}} \hat{u}_{\|}^{\phi_{2}} m^{\phi_{3}}
$$

A least-square fitting of the data against equation (24) yields the fitting coefficients:

$$
\phi_{1}=-0.89, \quad \phi_{2}=0.11, \quad \phi_{3}=-0.74
$$

The corresponding best fit plots are included in figures 10, 11, 12 and 13 as dashed lines.

\section{Conclusion and Discussion}

The identification of different mechanisms for cross flow beneath the gap motivated the independent analysis of their respective contributions. Despite the high level of abstraction, the derived sediment flow fractions and flux scaling relations provide a framework for assessing the effectiveness of silt screens for containing sediment spread. Buoyancy induced opposing cross flows beneath the screen have equal volume fluxes (due to continuity) which results in a turbid water flux to total exchange flux ratio of $0.5(\alpha=0.5)$. This can be conveniently characterized by the non-dimensional densimetric Froude number $F r \sim 0.5$ which has the opening distance $(\delta)$ and the density difference $\left(g^{\prime}\right)$ incorporated in it. It confirms that these two are the main mechanisms characterizing the flow 
beneath the screen. The density driven cross flow beneath a screen with different opening gaps is modelled by MIKE 3 Flow Model FM (the non-hydrostatic formulation) and similar results on flow characterization have been obtained, which confirms the accuracy of the identified driving mechanisms.

The screen motion induced sediment flux decreases as the screen gap increases, indicating declining mixing under the screen, towards the sea bed. Likewise, the induced sediment flux decreases with more oscillation modes. This can be attributed to the non-linear relation of the oscillation frequency and the oscillation mode. A slight increase of sediment flux was observed with increasing downstream flow. Summarizing, the screen motion induced cross flow is characterised through the maximum turbid water flow fraction $\alpha=0.5$ and the scaling relation:

$$
\hat{\jmath}_{\perp} \sim \hat{\delta}^{-0.89} \hat{u}_{\|}^{0.11} m^{-0.74}
$$

The flapping screen model could readily be applied for the analysis of the effectiveness of the silt screens under different flow conditions. Representing this in MIKE 3 Flow Model FM is done through a sub-model that exchange sediments using a diffusivity analogy. Furthermore, the effect of different screen materials or mooring strains on the ripping potential can be studied. Future work could also include a small-scale fluid-structure interaction model, yielding a better idea of the magnitude and dependency of the free parameters oscillation amplitude and wave length. Through these, the harmonic flapping model could be refined. Also, the effects of sedimentation induced seabed rise and tidal flows would complement the current models.

\section{Data Availability}

Some or all data, models, or code generated or used during the study are available from the corresponding author by request.

- Data:

- gravity current flow against volumetric sediment concentration, shown in Fig. 4.

- gravity current flow against silt screen gap width, shown in Fig. 5.

- screen motion induced flow against oscillation amplitude, shown in Fig. 9.

- screen motion induced flow against ambient current, shown in Fig. 10. 
- Models/Code:

- dynamic mesh model dynamicExplicitMotion and its implementation in OpenFOAM, see equations (20) and (21).

\section{Acknowledgements}

This work was supported by the Innovation Fund Denmark, Innovationsfonden, under the MEMENTO project with grant number 4106-00021B. DHI's office in Kota Kinabalu, Malaysia, are thanked for their guidance and experience sharing.

\section{References}

Aschmoneit, F. (2018). dynamicExplicitMotion. https://github.com/FynnAschmoneit/dynamicExplicitMotion. GitHub.

Benjamin, T. B. (1968). Gravity currents and related phenomena. Journal of Fluid Mechanics, 31(02), 209.

Chandrasekhar, S. (1981). Hydrodynamic and Hydromagnetic Stability (1st ed.). Oxford University Press.

Erftemeijer, P. L. A. \& Lewis, R. R. (2006). Environmental impacts of dredging on seagrasses: A review. Marine Pollution Bulletin, 52(12), 1553-1572.

Erftemeijer, P. L. A., Riegl, B., Hoeksema, B. W., \& Todd, P. A. (2012). Environmental impacts of dredging and other sediment disturbances on corals: A review. Marine Pollution Bulletin, 64(9), 1737-1765.

Fraser, M. W., Short, J., Kendrick, G., McLean, D., Keesing, J., Byrne, M., ... Wu, P. (2017). Effects of dredging on critical ecological processes for marine invertebrates, seagrasses and macroalgae, and the potential for management with environmental windows using Western Australia as a case study. Ecological Indicators, $78,229-242$.

Hjelmager Jensen, J. \& Saremi, S. (2014). Overflow Concentration and Sedimentation in Hoppers. Journal of Waterway, Port, Coastal, and Ocean Engineering, 140(6).

Hjelmager Jensen, J. \& Saremi, S. (2015). Effects of Mixing on Hopper Sedimentation in Clearing Mixtures. Journal of Waterway, Port, Coastal, and Ocean Engineering, 141(2).

JBF Scientific Corporation. (1978). An Analysis of the Functional Capabilities and Performance of Silt Curtains. 
Manela, A. \& Howe, M. S. (2009). On the stability and sound of an unforced flag. Journal of Sound and Vibration, $321(3), 994-1006$.

Michelin, S., Llewekkyn Smith, S. G., \& Glover, B. J. (2008). Vortex shedding model of a flapping flag. Journal of Fluid Mechanics, 617, 1-10.

Nayar, K. G., Sharqawy, M. H., Banchik, L. D., \& Lienhard, J. H. (2016). Thermophysical properties of seawater: A review and new correlations that include pressure dependence. Desalination, 390, 1-24.

OpenFOAM. (2019). Retrieved from https://openfoam.org/

Osipov, V. I. (2012). Density of Clay Minerals. Soil Mechanics and Foundation Engineering, 48(6), 231-240.

Pilarczyk, K. (2000). Geosynthetics and Geosystems in Hydraulic and Coastal Engineering (1st ed.). CRC Press.

Radermacher, M., de Wit, L., Winterwerp, J. C., \& Uijttewaal, W. S. J. (2016). Efficiency of Hanging Silt Curtains in Crossflow. Journal of Waterway, Port, Coastal, and Ocean Engineering, 142(1).

Research, Engineering and Development Center Vicksburg MS. (2005). Silt Curtains as a Dredging Project Management Practice. Retrieved from http://www.dtic.mil/dtic/tr/fulltext/u2/a437859.pdf

Roache, P. J. (1998). Verification and validation in computational science and engineering. Albuquerque, NM: Hermosa.

Vu, T. T. \& Tan, S. K. (2010). Laboratory investigation of hydraulic performance of silt screens. Journal of Hydrodynamics, Ser. B, 22(5, Supplement 1), 312-317.

WU, Y. S., Neo, E., Jain, M., \& Tan, C. A. (2016). Effectiveness of Silt Screen in Front of Industrial Water Intake. WODCON XXI Proceedings.

Yasui, A., Deguchi, I., \& Ono, M. (1999). Performance of Silt Protector In Three Dimensional Flow. Proceedings of the Ninth International Offshore and Polar Engineering Conference, 3. 


\section{${ }_{373} \quad 7 \quad$ Tables and Figures}

Table 1: Boundary conditions for Gravity Current Model. For boundary naming convention, see Fig. 2.

\begin{tabular}{llll}
\hline Boundary name & Velocity & Pressure & Silt concentration \\
\hline $\mathrm{X} 0, \mathrm{X} 1$ & $\partial_{x} u_{i}=0$ & $\partial_{x} P=0$ & $\begin{cases}\partial_{x} c=0, & \text { outflow } \\
c=c_{s}, & \text { inflow }\end{cases}$ \\
$\mathrm{Y} 0, \mathrm{Y} 1$ & $u_{i}=0$ & $\partial_{y} P=0$ & $\partial_{y} c=0$ \\
$\mathrm{SC}$ & $u_{i}=0$ & $\partial_{x} P=0$ & $\partial_{x} c=0$ \\
\hline
\end{tabular}


Table 2: Simulation parameters for gravity current model.

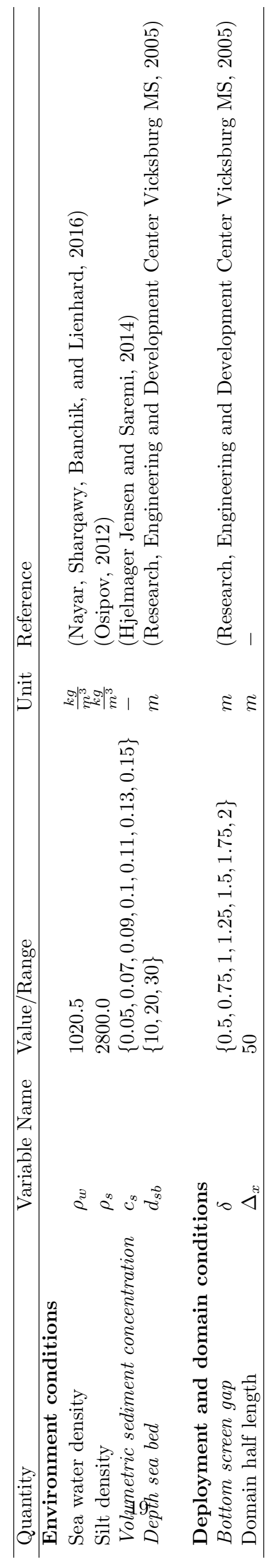


Table 3: Simulation parameters for screen flapping analysis. All test parameters are indicated through italic font.

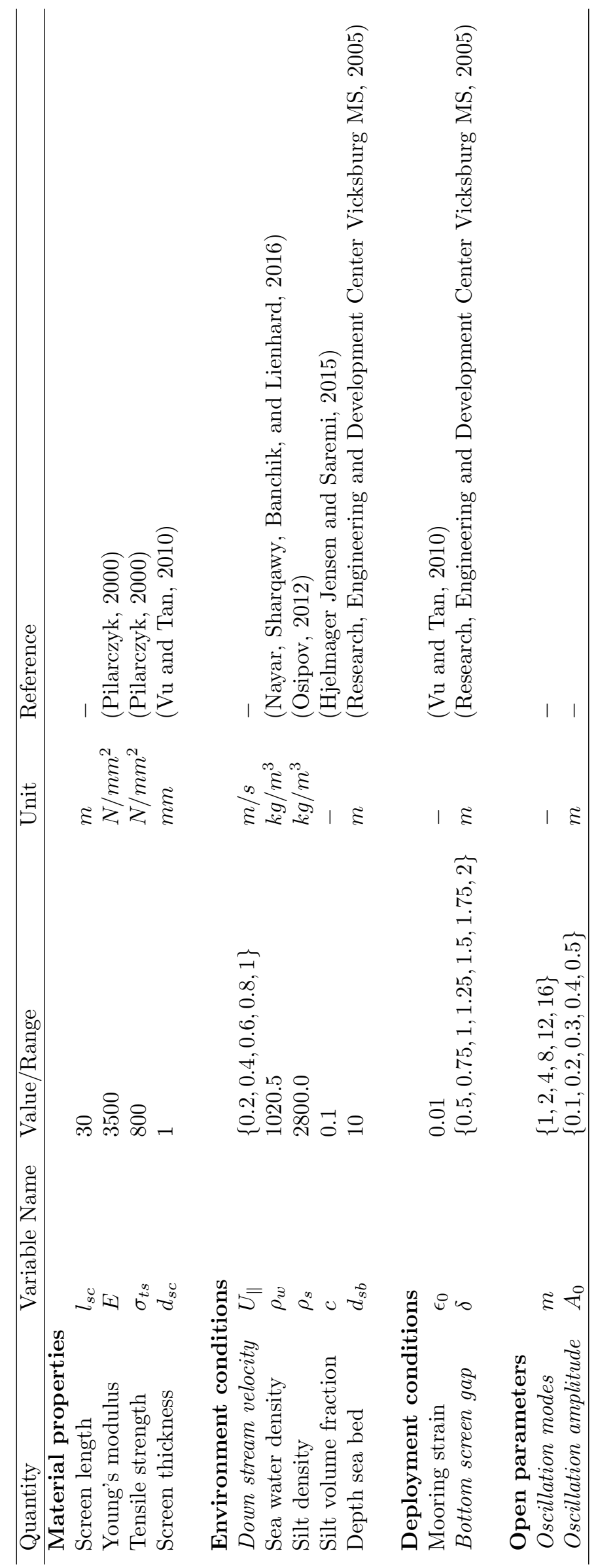


${ }_{375}$ Fig. 1: Floating silt screen separating a turbid water reservoir on the left-hand side from the sea water on the

${ }_{380}$ Fig. 2: Buoyancy induced flow of two opposing plumes. $d_{s b}, \delta$ and $\Delta_{x}$ denote the depth of the sea bed, the bottom screen gap, and the half-domain width, respectively. $\rho$ and $\rho_{w}$ denote the densities of the turbid- and sea water and $X 0, X 1, Y 0, Y 1, S C$ refer to the domain boundaries.

383Fig. 3: Snap shot of suspended sediment concentration field: The black arrows indicate the local flow direction. The turbid water creeping flow along the sea bed and the buoyant sea water plume on the left-hand side of the screen are clearly visible. The shear flow at the turbid water - sea water interface cause eddies, which are highlighted by the green ellipses.

${ }_{387}$ Fig. 4: The gravity current flow as a function of the volumetric sediment concentration for different screen gap heights with scaling law fit.

389Fig. 5: Gravity currents against screen gap heights for sediment concentrations and the limiting case of dissipationfree lock-exchange after (Benjamin, 1968).

391Fig. 6: Sediment mass flow rate per silt screen length against volumetric sediment concentration. The blue lines correspond to silt with a density of $2800 \mathrm{~kg} / \mathrm{m}^{3}$ and the red lines correspond to clay with a density of $1400 \mathrm{~kg} / \mathrm{m}^{3}$. The solid and dashed lines refer to screen gaps of $1 \mathrm{~m}$ and $0.5 \mathrm{~m}$, respectively.

394Fig. 7: Computational domain indicating domain dimensions and surface naming convention.

${ }_{395}$ Fig. 8: Streamline visualization in upstream perspective.

396Fig. 9: Time series of turbidity spread beneath the screen. The red color indicates a silt volume fraction of $c=0.1$. The blue color indicates fresh water. The exchange of sea water and fresh water in equal parts, and their resulting mixing, is clearly seen.

3\$ig. 10: Screen motion induced flow against oscillation amplitude with $\delta=1 \mathrm{~m}$.

44Fig. 11: Screen motion induced flow against downstream flow with $\delta=1 \mathrm{~m}$. 


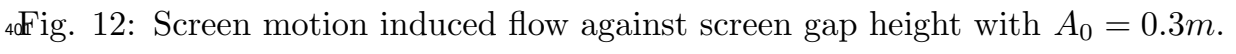

4dEig. 13: Screen motion induced flow against oscillation modes with $\delta=1 \mathrm{~m}$. 


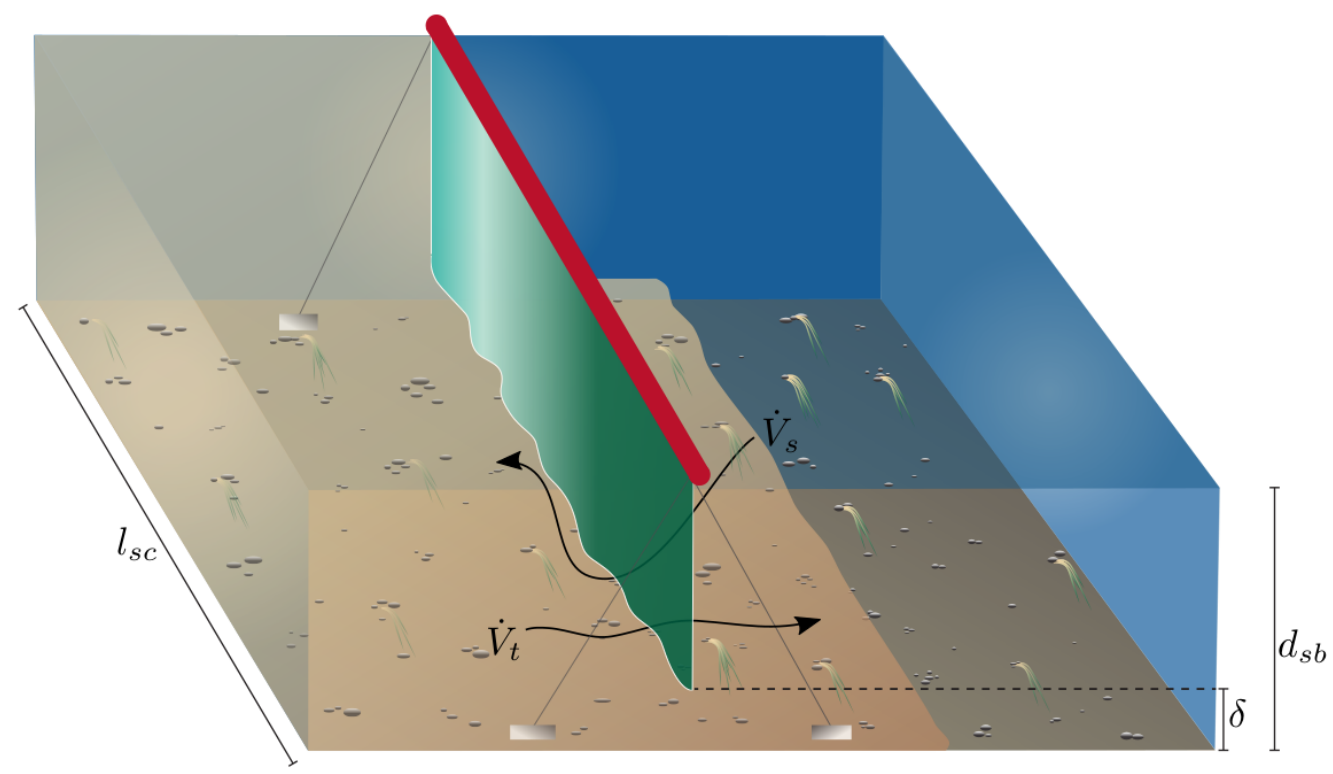

Figure 1: Floating silt screen separating a turbid water reservoir on the left-hand side from the sea water on the right-hand side. The screen is moored in the upstream plane and the downstream plane and hold up by the red float tube. The turbidity gradient causes a gravity current, which is indicated by the creeping flow of turbid water along the seabed. The silt screen element is of length $l_{s c}$. It leaves a gap of $\delta$ above the sea bed at depth $d_{s b}$.

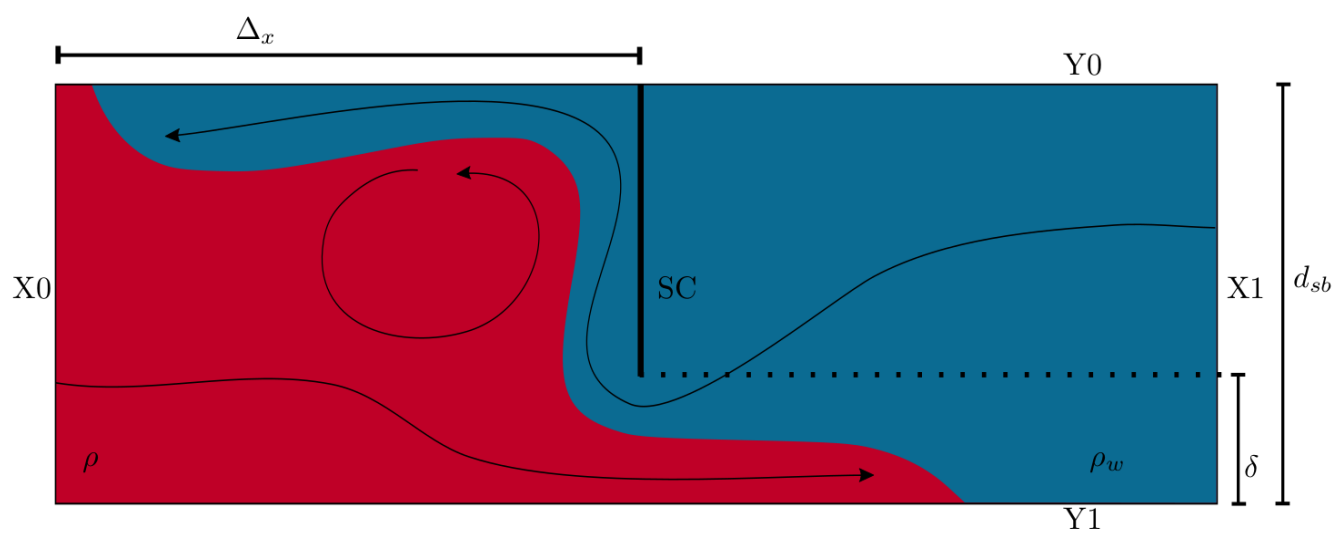

Figure 2: Buoyancy induced flow of two opposing plumes. $d_{s b}, \delta$ and $\Delta_{x}$ denote the depth of the sea bed, the bottom screen gap, and the half-domain width, respectively. $\rho$ and $\rho_{w}$ denote the densities of the turbid- and sea water and $X 0, X 1, Y 0, Y 1, S C$ refer to the domain boundaries. 


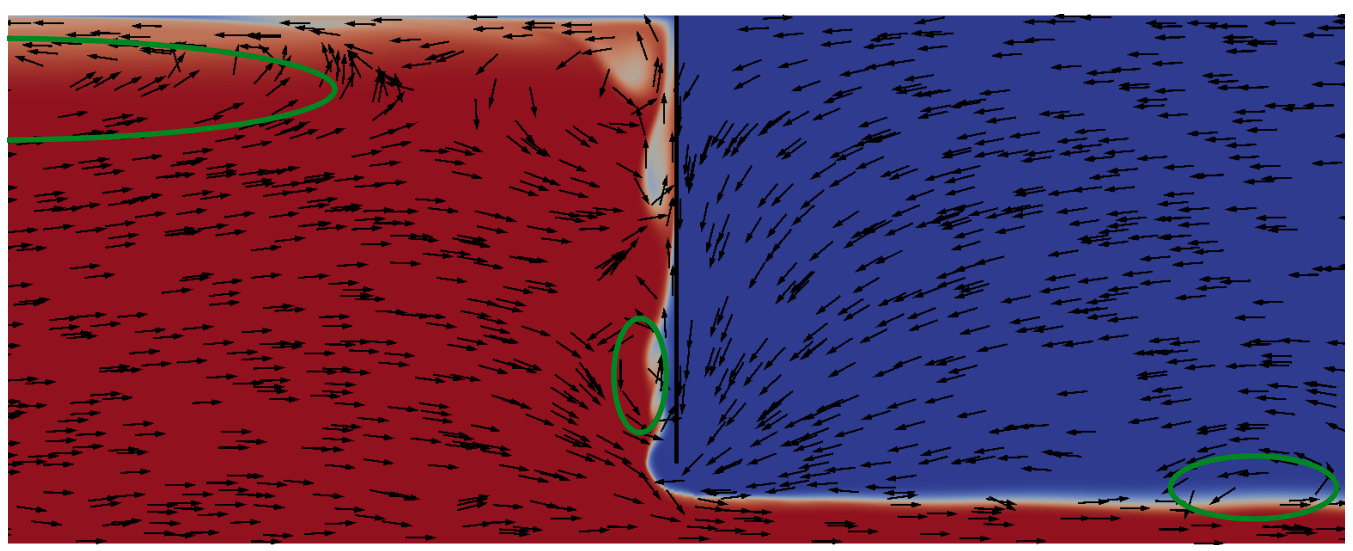

Figure 3: Snap shot of suspended sediment concentration field: The black arrows indicate the local flow direction. The turbid water creeping flow along the sea bed and the buoyant sea water plume on the left-hand side of the screen are clearly visible. The shear flow at the turbid water - sea water interface cause eddies, which are highlighted by the green ellipses.

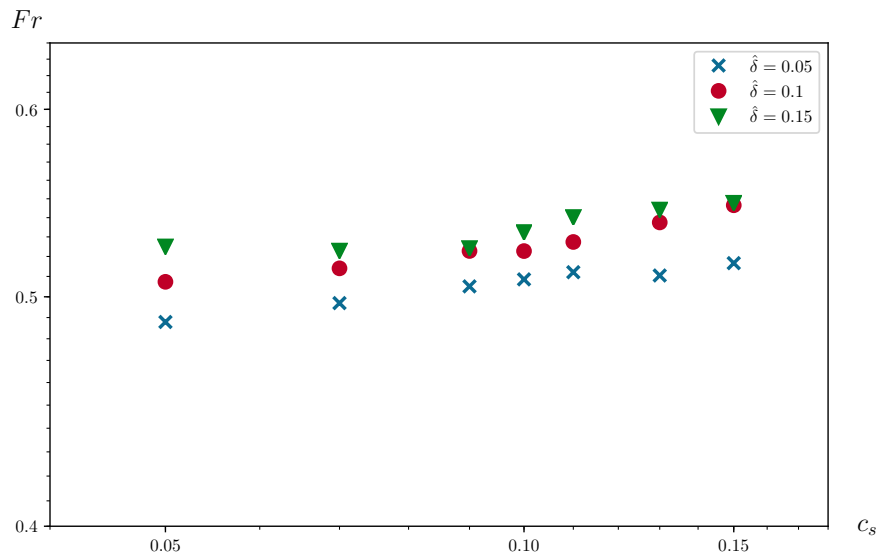

Figure 4: The gravity current flow as a function of the volumetric sediment concentration for different screen gap heights with scaling law fit.

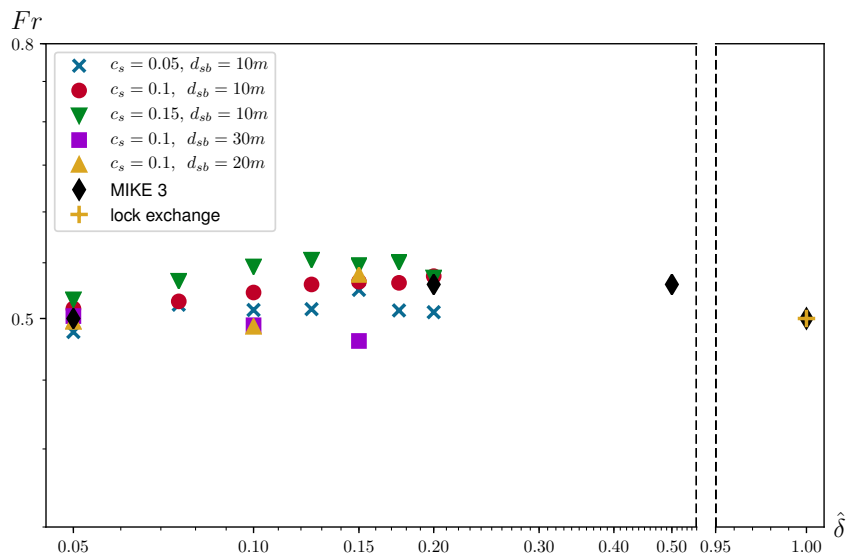

Figure 5: Gravity currents against screen gap heights for sediment concentrations and the limiting case of dissipation- free lock-exchange after (Benjamin, 1968). 


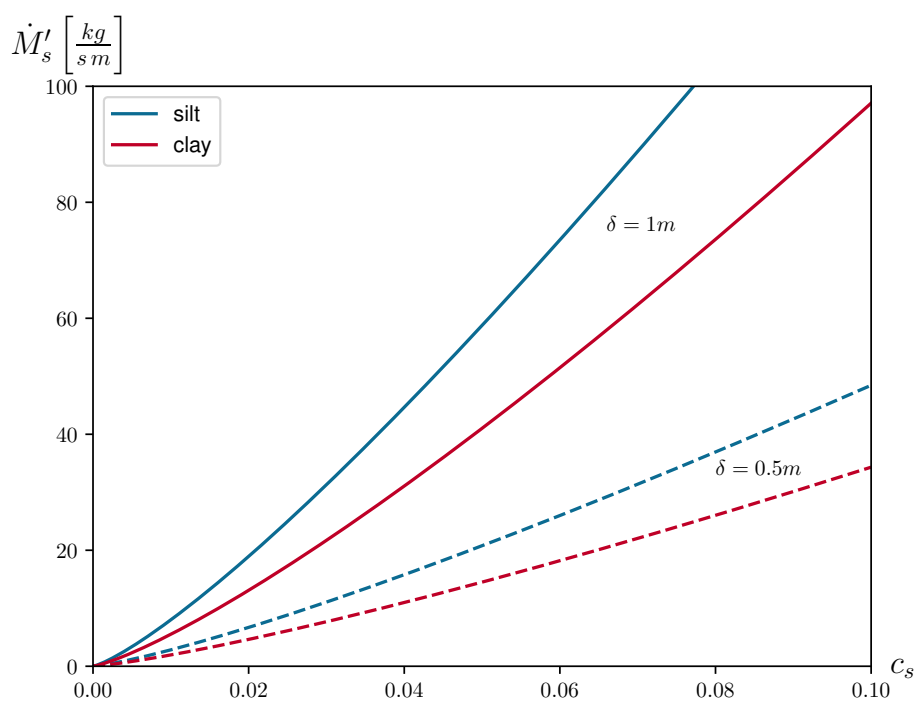

Figure 6: Sediment mass flow rate per silt screen length against volumetric sediment concentration. The blue lines correspond to silt with a density of $2800 \mathrm{~kg} / \mathrm{m}^{3}$ and the red lines correspond to clay with a density of 1400 $\mathrm{kg} / \mathrm{m}^{3}$. The solid and dashed lines refer to screen gaps of $1 \mathrm{~m}$ and $0.5 \mathrm{~m}$, respectively.

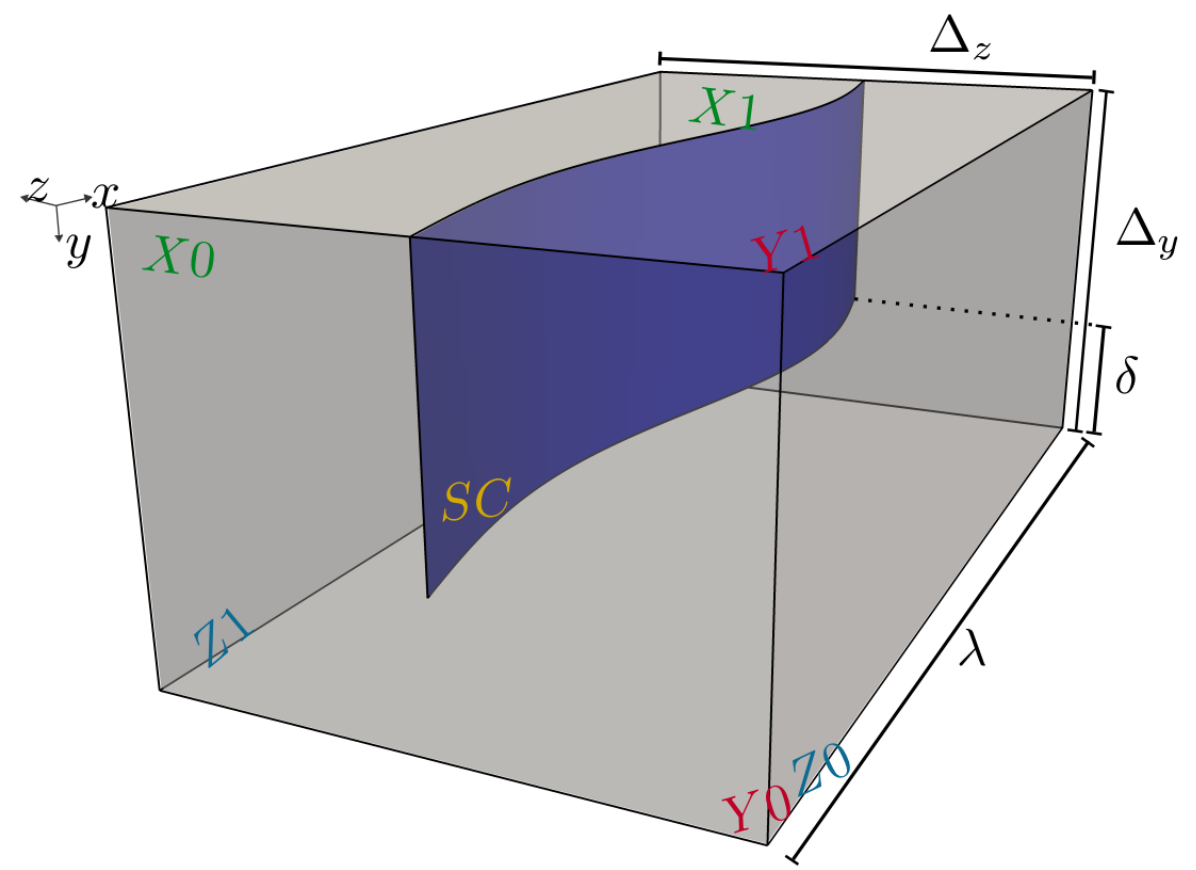

Figure 7: Computational domain indicating domain dimensions and surface naming convention. 


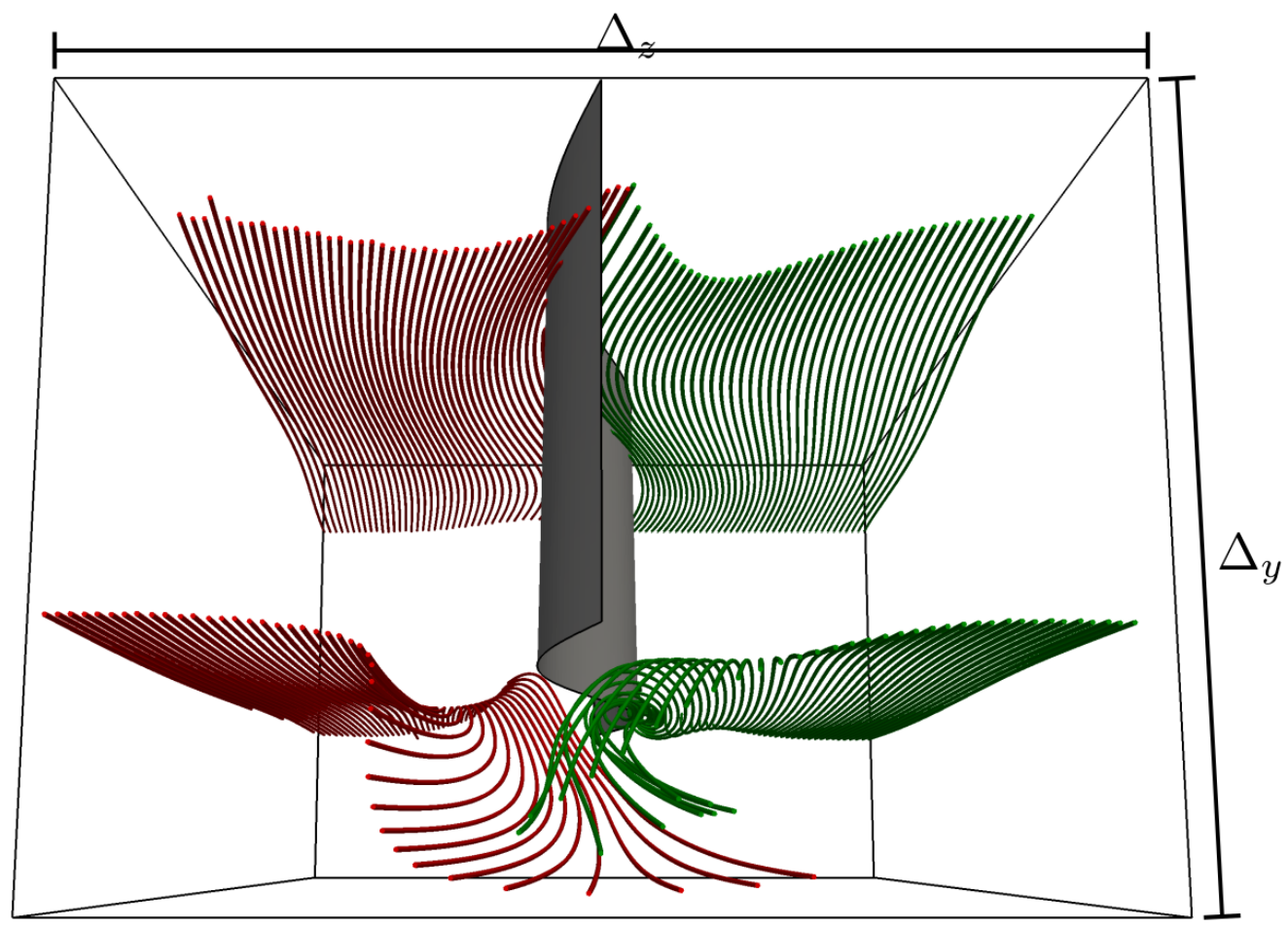

Figure 8: Streamline visualization in upstream perspective. 

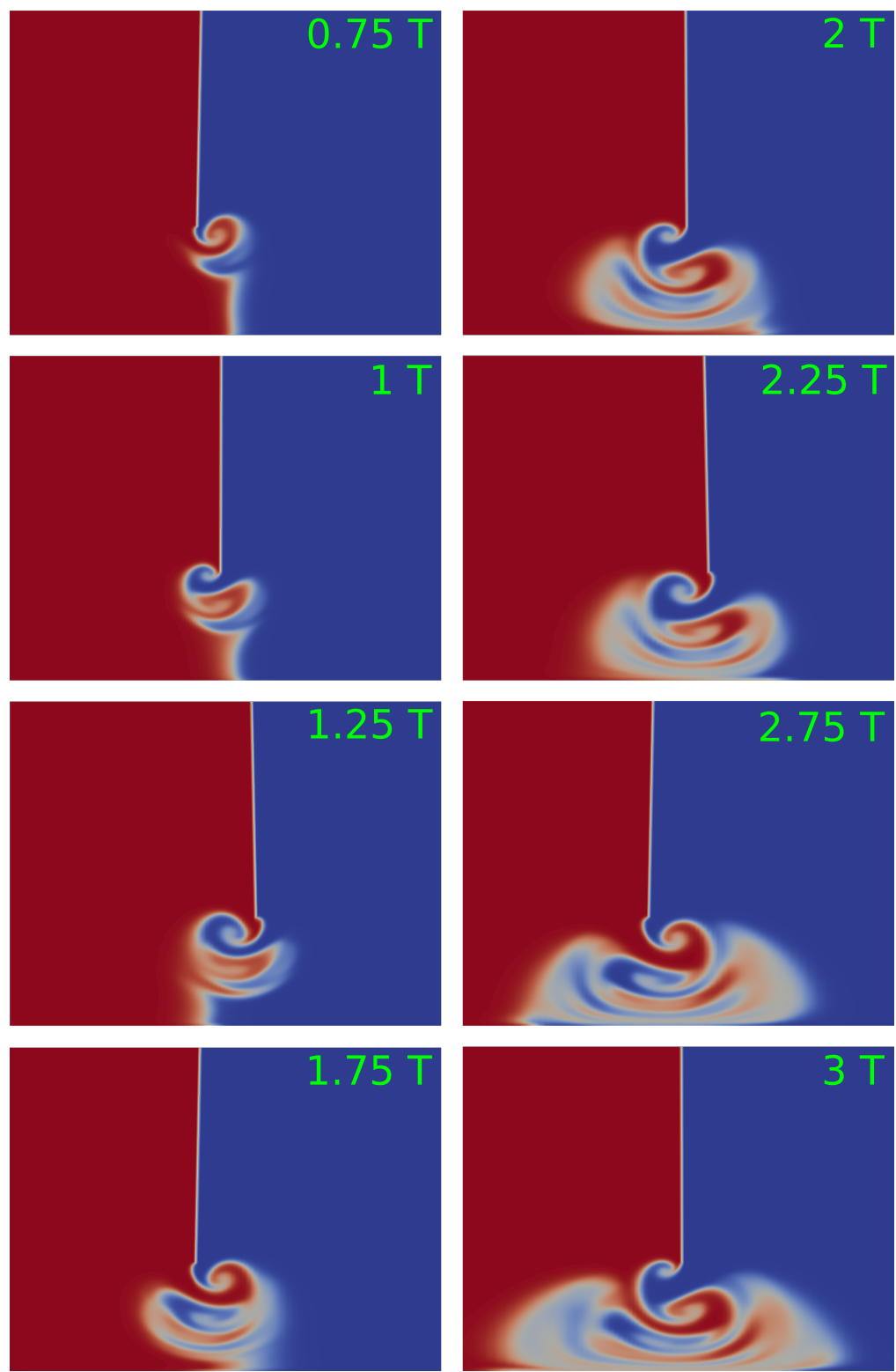

Figure 9: Time series of turbidity spread beneath the screen. The red color indicates a silt volume fraction of $c=0.1$. The blue color indicates fresh water. The exchange of sea water and fresh water in equal parts, and their resulting mixing, is clearly seen.

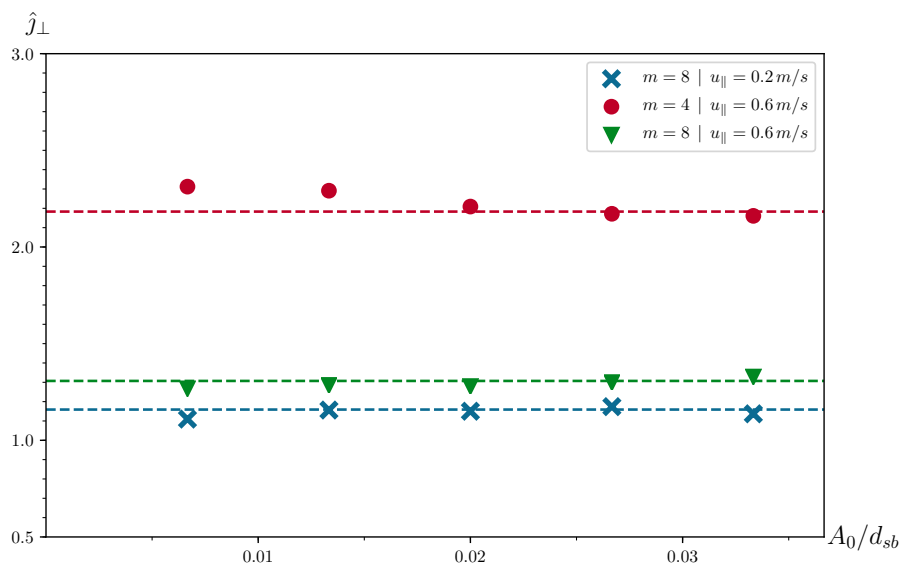

Figure 10: Screen motion induced flow against oscillation amplitude with $\delta=1 \mathrm{~m}$. 


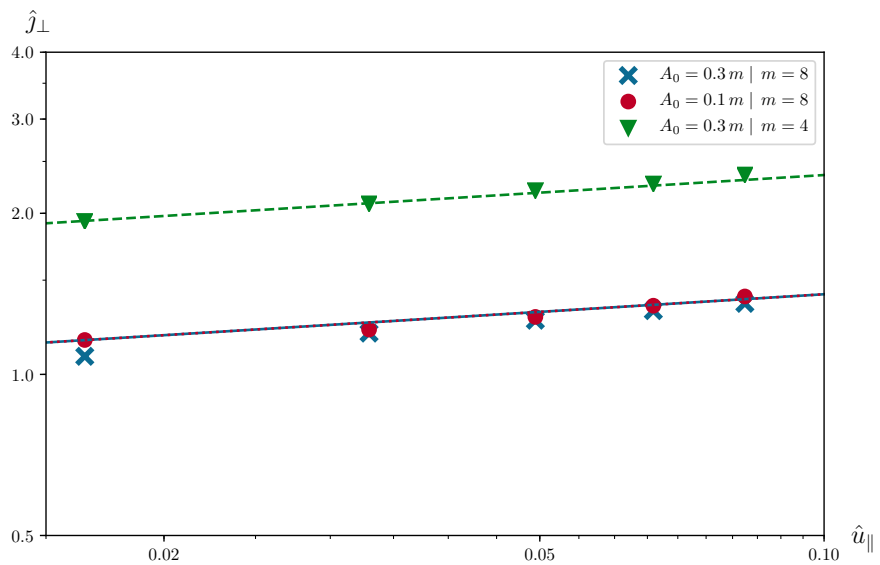

Figure 11: Screen motion induced flow against downstream flow with $\delta=1 \mathrm{~m}$.

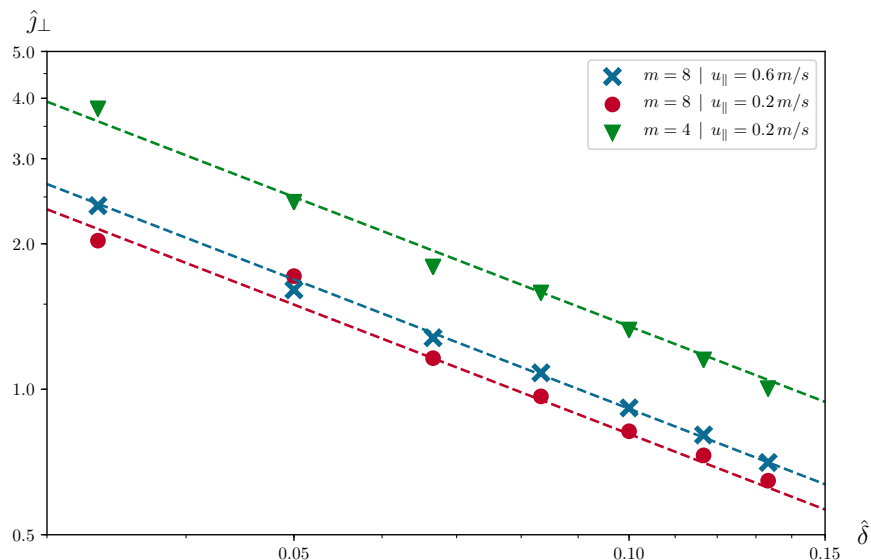

Figure 12: Screen motion induced flow against screen gap height with $A_{0}=0.3 \mathrm{~m}$.

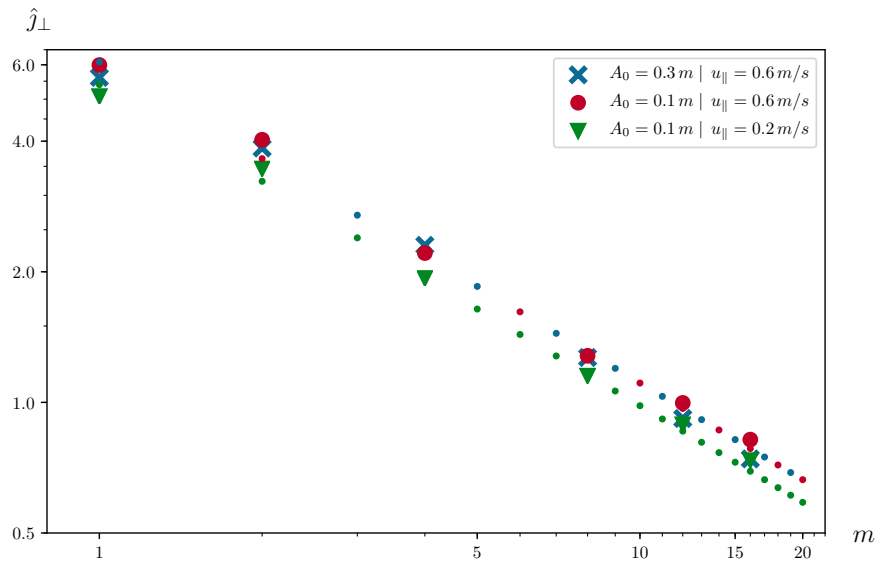

Figure 13: Screen motion induced flow against oscillation modes with $\delta=1 \mathrm{~m}$. 